\title{
MARKOV'S INEQUALITY FOR POLYNOMIALS WITH REAL ZEROS
}

\author{
PETER BORWEIN ${ }^{1}$
}

\begin{abstract}
Markov's inequality asserts that $\left\|p_{n}^{\prime}\right\| \leqslant n^{2}\left\|p_{n}\right\|$ for any polynomial $p_{n}$ of degree $n$. (We denote the supremum norm on $[-1,1]$ by $\|\cdot\|$.) In the case that $p_{n}$ has all real roots, none of which lie in $[-1,1]$, Erdös has shown that $\left\|p_{n}^{\prime}\right\| \leqslant e n\left\|p_{n}\right\| / 2$. We show that if $p_{n}$ has $n-k$ real roots, none of which lie in $[-1,1]$, then $\left\|p_{n}^{\prime}\right\| \leqslant c n(k+1)\left\|p_{n}\right\|$, where $c$ is independent of $n$ and $k$. This extension of Markov's and Erdös' inequalities was conjectured by Szabados.
\end{abstract}

Introduction. Markov's inequality asserts that

$$
\left\|p_{n}^{\prime}\right\|_{[-1,1]} \leqslant n^{2}\left\|p_{n}\right\|_{[-1,1]}
$$

for any poynomial $p_{n} \in \pi_{n}$ [2 and 3]. ( $\pi_{n}$ denotes the algebraic polynomials of degree at most $n$ and $\|\cdot\|_{A}$ denotes the supremum norm on $A$.) Erdös [1] in 1940 offered the following refinement of Markov's inequality. If $p_{n} \in \pi_{n}$ and $p_{n}$ has all its roots in $\mathbf{R}-(-1,1)$, then

$$
\left\|p_{n}^{\prime}\right\|_{[-1,1]} \leqslant \frac{e n}{2}\left\|p_{n}\right\|_{[-1,1]} .
$$

Inequality (1) iterates to give bounds for the $k$ th derivative of a polynomial. However, we cannot proceed inductively with inequality (2) since some of the roots of the derivatives may be in $[-1,1]$. With this in mind, Szabados and Varma established a version of (2) for polynomials of degree $n$ with all real roots and at most one root in $[-1,1]$, namely, for such a polynomial $p_{n}$,

$$
\left\|p_{n}^{\prime}\right\|_{[-1,1]} \leqslant c_{1} n\left\|p_{n}\right\|_{[-1,1]},
$$

where $c_{1}$ is independent of $n$ [5]. This, of course, yields the following inequality:

$$
\left\|p_{n}^{\prime \prime}\right\|_{[-1,1]} \leqslant c_{2} n^{2}\left\|p_{n}\right\|_{[-1,1]}
$$

for any $p_{n} \in \pi_{n}$ that has all its roots in $\mathbf{R}-(-1,1)$. In [6] Szabados proposed the following

Conjecture. If $p_{n}$ is a polynomial of degree $n$ and $p_{n}$ has at least $n-k$ roots in $\mathbf{R}-(-1,1)$, then there is a constant $c(c \leqslant 9)$ so that

$$
\left\|p_{n}^{\prime}\right\|_{[-1,1]} \leqslant c n(k+1)\left\|p_{n}\right\|_{[-1,1]} .
$$

Received by the editors November 28, 1983.

1980 Mathematics Subject Classification. Primary 26C05, $26 \mathrm{C} 10$.

${ }^{1}$ Research supported, in part, by N.S.E.R.C. of Canada. 
It is our intention to prove this slightly strengthened form of Szabados' conjecture. In its original form the conjecture had the additional assumption that all the roots of $p_{n}$ be real. Up to the constant this result is best possible; Szabados in [6] constructs polynomials $p_{n}$ of degree $n$ with $n-k$ roots in $\mathbf{R}-(-1,1)$ so that

$$
\left\|p_{n}^{\prime}\right\|_{[-1,1]} \geqslant \frac{n \cdot k}{2}\left\|p_{n}\right\|_{[-1,1]} \quad(0<k \leqslant n) .
$$

It is apparent from (1) and (2) that the best constant must depend on $k$. Some related results may be found in [4].

Inequalities for the higher derivatives of polynomials with real roots can now be derived straightforwardly from (5). For example,

THEOREM. If $p_{n} \in \pi_{n}$ has at least $n-k$ zeros in $\mathbf{R}-(-1,1)$, then

$$
\left\|p_{n}^{(m)}\right\|_{[-1,1]} \leqslant c_{m} \frac{n !(k+m) !}{(n-m) ! k !}\left\|p_{n}\right\|_{[-1,1]},
$$

where $c_{m} \leqslant 9^{m}$ depends only on $m$.

2. Proof of the Conjecture. Let $c_{2 k}$ be the $2 k$ th Chebychev polynomial shifted to the interval [0,2] and normalized to have lead coefficient 1 . Let $\alpha_{1}<\alpha_{2}<\cdots<\alpha_{k}$ be the roots of $c_{2 k}$ in $[1,2]$ and let

$$
t_{k}:=\prod_{i=1}^{k}\left(x-\alpha_{i}\right)
$$

LEMMA 1. The polynomial $q_{k}:=(x+2 m / k)^{m+k} t_{k}(x)$ has the following property. If $\alpha_{0}=0$ and $\alpha_{n+1}=1$, then, for $i=1,2, \ldots, n$,

$$
\left\|\left(x+\frac{2 m}{k}\right)^{m+k} t_{k}\right\|_{\left[\alpha_{i-1}, \alpha_{1}\right]}>\left\|\left(x+\frac{2 m}{k}\right)^{m+k} t_{k}\right\|_{\left[\alpha_{i}, \alpha_{i+1}\right]},
$$

where the maximums on successive intervals occur with alternating sign.

Proof. Let $0<\beta_{1}<\cdots<\beta_{k}$ be the roots of $c_{2 k}$ in $[0,1]$. We observe that $x /\left(x-\beta_{i}\right)$ is positive and decreasing on $\left(\beta_{k}, \infty\right]$ and that $c_{2 k}$ equioscillates on the intervals in question (i.e. $c_{2 k}$ satisfies (7) with equality). We now note that

$$
x^{k} t_{k}=\left(\prod_{i=1}^{k} \frac{x}{\left(x-\beta_{i}\right)}\right) c_{2 k}
$$

satisfies the conclusion of Lemma 1 . To finish the proof we need only observe that $(x+2 m / k)^{m+k} / x^{k}$ is decreasing on $[0,2]$.

Let $n=2 k+m$ and let

$$
s_{n}(x):=\frac{1}{(1+m / k)^{n}} q_{k}\left(\left(1+\frac{m}{k}\right) x+\left(1-\frac{m}{k}\right)\right) .
$$

(We have shifted from $[-2 m / k, 2]$ to $[-1,1]$.) This polynomial will act as a kind of near extremal polynomial for the Conjecture. Let $\gamma_{1}<\gamma_{2}<\cdots<\gamma_{k}$ be the roots of $s_{n}$ in $(-1,1)$. We collect the properties of $s_{n}$ that we require in the next lemma. 
LEMMA 2. For $n=m+2 k$ and $s_{n}$ as above:

(a) $s_{n}(x)=(x+1)^{m+k} \Pi_{i=1}^{k}\left(x-\gamma_{i}\right)$,

(b) $\sum_{i=1}^{k}\left(1 /\left(1-\gamma_{i}\right)\right) \leqslant 4 k(n-k)$, and

(c) for $i=1, \ldots, k, \gamma_{0}=-1$ and $\gamma_{k+1}=1$

$$
\left\|s_{n}\right\|_{\left[\gamma_{i-1}, \gamma_{i}\right]} \geqslant\left\|s_{n}\right\|_{\left[\gamma_{i}, \gamma_{i+1}\right]} \text {. }
$$

Proof. Parts (a) and (c) are immediate from the construction of $s_{n}$. Part (b) follows from the observation that, for $\alpha_{i}$ as in (6).

$$
\sum_{i=1}^{k} \frac{1}{2-\alpha_{i}} \leqslant \frac{c_{2 k}^{\prime}(2)}{c_{2 k}(2)}=4 k^{2}
$$

and the observation that

$$
1-\gamma_{i}=\frac{1}{1+m / k}\left(2-\alpha_{i}\right)
$$

Let $p_{n}^{*} \in \pi_{n}$ maximize

$$
\left|p_{n}^{\prime}(1)\right| /\left\|p_{n}\right\|_{[-1,1]},
$$

where the maximum is taken over all polynomials in $\pi_{n}$ that have all but at most $k$ roots in $\mathbf{R}-(-1,1)$. The information we need about $p_{n}^{*}$ is contained in the next lemma.

LeMma 3. Let $p_{n}^{*}$ be as above. Then

(a) $p_{n}^{*}$ has $k$ simple roots $\delta_{1}<\cdots<\delta_{k}$ in $(-1,1), p_{n}^{*}$ has $n-k$ roots at \pm 1 , and $p_{n}^{*}$ achieves its maximum modulus on each of the intervals $\left[-1, \delta_{1}\right],\left[\delta_{1}, \delta_{2}\right], \ldots,\left[\delta_{k}, 1\right]$.

(b) Either $p_{n}^{*}$ has no roots in $[-1, \infty)$ or $p_{n}^{*}$ has exactly one root at 1 .

PROOF. The proof of (a) is a simple and standard perturbation argument (if $p_{n}^{*}$ did not satisfy (a) then it would be possible to perturb $p_{n}^{*}$ to reduce its norm on $[-1,1]$ without decreasing the derivative at 1$)$. We will prove only that $p_{n}$ has no roots in $(1, \infty)$, the other parts are similar. First suppose that $p_{n}^{*}$ has two roots at $\alpha>1$ and $\beta>1$. Consider

$$
v_{n}(x):=\frac{p_{n}^{*}(x)(x-1)^{2}}{(x-\alpha)(x-\beta)} .
$$

Then for sufficiently small $\varepsilon>0$ :

(i) $\left\|p_{n}^{*}-\varepsilon v_{n}\right\|_{[-1,1]}<\left\|p_{n}^{*}\right\|_{[-1,1]}$,

(ii) $\left|\left(p_{n}^{*}-\varepsilon v_{n}\right)^{\prime}(1)\right|=\left|p_{n}^{* \prime}(1)\right|$,

(iii) $p_{n}^{*}-\varepsilon v_{n}$ has all but at most $k$ roots in $\mathbf{R}-(1,1)$.

Part (iii) follows since $(x-\alpha)(x-\beta)-\varepsilon(x-1)^{2}$ has two roots in $[1, \infty]$ for sufficiently small $\varepsilon$. (Note $\alpha$ may equal $\beta$.) However, this contradicts the maximality of $p_{n}^{*}$.

Next we suppose that $p_{n}^{*}$ has exactly one (nonrepeat) root at $\alpha>1$. Now we argue as before by considering

$$
v_{n}(x):=p_{n}^{*}(x) \frac{(x-1)^{2}}{(x-1)(x-\alpha)}
$$


If $p_{n}^{*}(1) \neq 0$ we must observe that in this case $\operatorname{sign}\left(p_{n}^{*}(1)\right)=\operatorname{sign}\left(p_{n}^{* \prime}(1)\right)$ and, hence, that

$$
v_{n}^{\prime}(1)=\frac{(1-\alpha) p_{n}^{*}(1)}{(1-\alpha)^{2}}
$$

has the opposite sign to $p_{n}^{* \prime}(1)$. The last observation requires noticing that if $p_{n}^{* \prime}(1)$ has opposite sign to $p_{n}^{*}(1)$, then $p_{n}^{* \prime}$ has all its zeros in $(-\infty, 1]$ and, hence, $p_{n}^{\prime \prime}(1) \neq 0$. Thus, $\left|p_{n}^{* \prime}\right|$ is increasing on $[1, \infty),\left|p_{n}\right|$ is decreasing on $[1, \alpha)$ and $p_{n}^{*}(x+(\alpha-1))$ violates the maximality assumptions on $[-1,1]$.

Part (b) follows since if $p_{n}^{*}$ has two or more zeros at 1 , then $p_{n}^{\prime}(1)$ would also equal zero.

LEMMA 4. If $p_{n} \in \pi_{n}$ has at least $(n-k)$ roots in $\mathbf{R}-(-1,1)$, then

$$
\left|p_{n}^{\prime}(1)\right| \leqslant \frac{9}{2}(k+1) n\left\|p_{n}\right\|_{[-1,1]} \text {. }
$$

Proof. If $2 k \geqslant n$, then the lemma follows from Markov's inequality, so we may suppose $2 k<n$. Suppose there exists $p_{n}$, as above, so that

$$
\left|p_{n}^{\prime}(1)\right|>\frac{9}{2}(k+1)(n-k)\left\|p_{n}\right\|_{[-1,1]},
$$

and let $q_{n}$ be the maximal such $p_{n}$. By Lemma 3, this $q_{n}$ equioscillates $k+1$ times on $[-1,1]$.

We shall first consider the case where $q_{n}$ has no root at 1 .

The key to the proof is to observe that the roots of $q_{n}$ lie to the left of the roots of $s_{n}$ (as defined in Lemma 3). We may write

$$
q_{n}(x)=(x+1)^{n-k} \prod_{i=1}^{k}\left(x-\rho_{i}\right),
$$

where $-1<\rho_{1}<\cdots<\rho_{k}<1$. Also,

$$
s_{n}(x)=(x+1)^{n-k} \prod_{i=1}^{k}\left(x-\gamma_{i}\right) \text {. }
$$

The claim is that $\gamma_{i} \geqslant \rho_{i}$ for each $i$. This is seen as follows.Choose the largest $i$ for which $\rho_{i}>\gamma_{i}$. Then pick $\eta$ so that $\left\|\eta q_{n}\right\|_{\left[\gamma_{i}, 1\right]}=\left\|s_{n}\right\|_{\left[\gamma_{i}, 1\right]}$. (We will specify the sign of $\eta$ later.)We can deduce from the equioscillation of $q_{n}$ that $\eta q_{n}-s_{n}$ has at least $k-i$ roots on $[\beta, 1]$, where $\beta$ is the first point greater than $\rho_{i}$ where $\eta q_{n}$ achieves its maximum modulus. From Lemma 2(c) we deduce that $\eta q_{n}-s_{n}$ has at least $i-1$ roots on $(-1, \alpha)$, where $\alpha$ is the largest point less than $\gamma_{i}$ where $s_{n}$ achieves its maximum modulus. We need only observe that if we choose the sign of $\eta$ so that

$$
\operatorname{sign} \eta q_{n}(\beta)=-\operatorname{sign} s_{n}(\alpha),
$$

then $\eta q_{n}-s_{n}$ must have 2 roots in $(\alpha, \beta)$. Thus, $\eta q_{n}-s_{n}$ has $n+1$ roots which is a contradiction and we conclude that $\rho_{i} \leqslant \gamma_{i}$. 
We now observe that, since $\rho_{i} \leqslant \gamma_{i}<1$,

$$
\begin{aligned}
\frac{\left|q_{n}^{\prime}(1)\right|}{\left\|q_{n}\right\|_{[-1,1]}} & =\frac{q_{n}^{\prime}(1)}{q_{n}(1)}=\sum_{i=1}^{k} \frac{1}{1-\rho_{i}}+\sum_{i=1}^{n-k} \frac{1}{1-(-1)} \\
& \leqslant \sum_{i=1}^{k} \frac{1}{1-\gamma_{i}}+\frac{n-k}{2} \leqslant 4 k(n-k)+\frac{n-k}{2},
\end{aligned}
$$

where the later inequality follows from Lemma 2 . This is a contradiction.

In the case where $q_{n}$ has exactly one root at 1 we proceed as follows. Let $d>1$ be the unique point in $(1, \infty)$, where $\left|q_{n}(d)\right|=\left\|q_{n}\right\|_{[-1,1]}$. We can now consider $q_{n}$ on $[-1, d]$. We note that

$$
\frac{\left|q_{n}^{\prime}(d)\right|}{\left\|q_{n}\right\|_{[-1,1]}} \geqslant \frac{\left|q_{n}^{\prime}(1)\right|}{\left\|q_{n}\right\|_{[-1,1]}}
$$

since $\left|q_{n}^{\prime}\right|$ is increasing on $[1, \infty)$. We can repeat verbatim the argument of the first part applied to

$$
\tilde{q}(x)=q_{n}\left(x\left(\frac{d+1}{2}\right)+\left(\frac{d-1}{2}\right)\right)
$$

with $k$ replaced by $k+1$. This allows us to deduce the contradiction that

$$
\frac{\left|q^{\prime}(1)\right|}{\left\|q_{n}\right\|_{[-1,1]}} \leqslant \frac{\left|\tilde{q}_{n}(1)\right|}{\left\|\tilde{q}_{n}\right\|_{[-1,1]}} \leqslant 4(k+1)(n-k)+\frac{n-k}{2} .
$$

The proof of the Conjecture is now straightforward.

Proof of Conjecture. Let $p_{n}$ be a polynomial of degree $n$ with $n-k$ roots in $\mathbf{R}-(-1,1)$. Let $x_{0}$ be a point in $[-1,1]$, where $p_{n}^{\prime}$ achieves its maximum modulus. We suppose $x_{0} \leqslant 0$ ( $x_{0}>0$ follows analogously). Let $a x+b$ map [ $\left.x_{0}, 1\right]$ one-to-one onto $[-1,1]$ in such a way that $x_{0} \rightarrow 1$.

Note that $|a|<2$. Thus, if $v_{n}(a x+b)=p_{n}(x)$, then

$$
\frac{\left|p_{n}^{\prime}\left(x_{0}\right)\right|}{\left\|p_{n}\right\|_{[-1,1]}} \leqslant \frac{2\left|v_{n}^{\prime}(1)\right|}{\left\|v_{n}\right\|_{[-1,1]}}=9 n(k+1),
$$

where the last inequality follows from Lemma 4 and the observation that $v_{n}$ has at least as many roots as $p_{n}$ in $\mathbf{R}-[-1,1]$.

\section{REFERENCES}

1. P. Erdös, On extremal properties of the derivatives of polynomials, Ann. of Math. (2) 41 (1940), 310-313.

2. G. G. Lorentz, Approximation of functions, Holt, Reinhart and Winston, New York, 1966.

3. A. A. Markov, On a problem of D. I. Mendeleev, Acad. Sci., St. Petersburg 62 (1889), 1-24.

4. A. Máté, Inequalities for derivatives of polynomials with restricted zeros, Proc. Amer. Math. Soc. 88 (1981), 221-225.

5. J. Szabados and A. K. Varma, Inequalities for derivatives of polynomials having real zeros, Approximations Theory. III (E. W. Cheney, ed.), Academic Press, New York, 1980, pp. $881-888$.

6. J. Szabados, Bernstein and Markov type estimates for the derivative of a polynomial with real zeros, Functional Analysis and Approximation (P. L. Butzer, B. Sz.-Nagy and E. Görlick, eds.), Birkhäuser, Basel, 1981, pp. 177-188.

Department of Mathematics, Statistics and Computing Science, Dalhousie University, Halifax, Nova Scotia B3H 4H8, Canada 